\title{
Capacity and Energy Efficiency of Multi-User Spectrum Sharing Systems with Opportunistic Scheduling
}

\author{
Tae Won Ban, Student Member, IEEE, Wan Choi, Member, IEEE, \\ and Dan Keun Sung, Senior Member, IEEE
}

\begin{abstract}
This paper investigates the capacity and energy efficiency of spectrum sharing systems with opportunistic user selection where a secondary network utilizes spectrum bands licensed to a primary network under interference regulation. In spectrum sharing systems, secondary users consume a fraction of their resources in sensing the channels to the primary users to comply with the interference constraints. Although more resources for sensing improve reliability and performance, the throughput loss due to time overhead and energy loss due to power overhead should be properly incorporated in performance evaluation. In this context, we define and derive a new metric average capacity normalized by the total energy consumption - reflecting time and power overhead for spectrum sensing. Based on the developed framework, the optimal normalizedcapacity is investigated. We also propose a simple and practical suboptimal best- $n$ scheme motivated by the infeasibility and high computational complexity of the optimal strategy, where $n$ denotes the number of sensing secondary users. Our analytical and simulation results show that the proposed best- 1 scheme is an energy-efficient technique with near optimality in terms of the capacity normalized by the energy consumption.
\end{abstract}

Index Terms-Spectrum sharing, cognitive radio, sensing power, multiuser diversity.

\section{INTRODUCTION}

$\mathbf{S}$ PECTRUM sharing has been recognized as one of promising techniques since it can greatly improve spectral utilization [1]-[3]. In spectrum sharing systems, secondary users utilize spectrum bands originally allocated to primary users under a regulation that the interference caused by secondary users at each primary user must be maintained below a predetermined level, i.e., interference temperature. The primary users are able to stably communicate with each other unless the perceived interference exceeds the interference temperature. While the previous studies on spectrum sharing systems focused on a single secondary user [4]-[6], an extension to multiple secondary users raises several interesting issues related to medium access control. In our previous studies, we analyzed the capacity of a secondary spectrum sharing system for three different power control schemes assuming perfect spectrum sensing without additional energy consumption [7]

Manuscript received July 16, 2008; revised December 11, 2008; accepted February 7, 2009. The associate editor coordinating the review of this letter and approving it for publication was M. Guizani.

The authors are with the School of Electrical Engineering and Computer Science, Korea Advanced Institute of Science and Technology, Daejeon, 305-701, Korea (e-mail: twban@cnr.kaist.ac.kr; wchoi@ee.kaist.ac.kr; dksung@ee.kaist.ac.kr).

This research was supported in part by the Center for Cooperative Wireless Communication through the ITRC support program supervised by the IITA (IITA-2009-C1090-0902-0005).

Digital Object Identifier 10.1109/TWC.2009.080944 and it was shown that multiuser diversity gain with opportunistic scheduling under interference regulation shows different scaling law from the case without spectrum sharing [8].

In spectrum sensing systems, secondary users consume a fraction of their available resources in spectrum sensing so that their available resources for data transmission are reduced [9][11]. Longer and more frequent spectrum sensing improves the accuracy and reliability of sensing at the sacrifice of throughput. Cabric et al. [9] numerically measured the probability of detection for varying sensing durations. Ghasemi and Sousa [10] showed that there exists an optimum sensing duration maximizing the spectral efficiency of a secondary user. Zhao et al. [11] presented an optimal spectrum access strategy using periodic channel sensing. In addition to the throughput loss due to the time overhead, spectrum sensing consumes secondary users' power and, hence, energy efficiency for data transmission decreases as the number of primary users increases. The potential of spectrum sharing systems needs to be precisely evaluated by considering both energy efficiency and achievable throughput, but the energy loss due to spectrum sensing has not been well incorporated in previous research.

In this paper, we define and derive achievable average capacity normalized by the energy consumption to reflect both time and power overhead due to spectrum sensing in evaluating the achievable capacity. In our spectrum sharing model, multiple primary receivers impose their constraints of interference temperature on secondary transmitters and a secondary receiver opportunistically selects a secondary transmitter for transmission among multiple secondary transmitters not causing interference above the interference temperature. Based on the developed formulation, we numerically evaluate the optimal average capacity normalized by the energy consumption where the effect of resource overhead due to spectrum sensing is reflected. Achieving the optimal normalized capacity is, however, practically infeasible since it requires a prior knowledge of interference channels for each secondary transmitter not in the optimal sensing set. Furthermore, the computational complexity in achieving the optimal normalized capacity increases as the number of secondary transmitter increases. Thus, we propose a simple and practical suboptimal scheme and compare the achievable normalized capacity of the the proposed scheme with that of the optimal scheme. Our analytical and numerical results show that the proposed suboptimal scheme is a practical energy-efficient technique with near optimality.

The rest of this paper is organized as follows. In Section II, a system model is described. In Section III, a capacity 


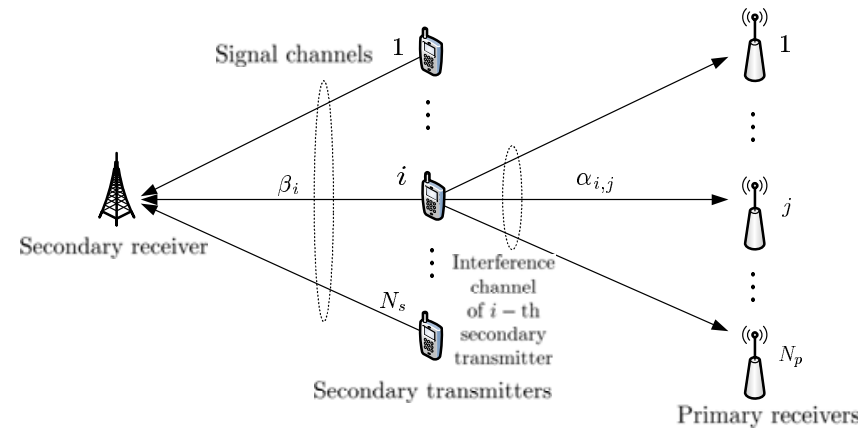

Fig. 1. System model

analysis problem is reformulated to reflect two different types of resource overhead due to spectrum sensing. The optimal capacity is derived based on the formulation. In Section IV, a suboptimal scheme is proposed. Section V presents numerical and simulation results. Finally, conclusions are drawn in Section VI.

\section{System Model}

Fig. 1 shows a spectrum sharing system with $N_{p}$ primary receivers and $N_{s}$ secondary transmitters. The channel gains $\alpha_{i, j}$ and $\beta_{i}$ denote the instantaneous gains of an interference channel from the $i$-th secondary transmitter to the $j$-th primary receiver and a signal channel from the $i$-th secondary transmitter to the secondary receiver, respectively. All the channel gains are independent and identically distributed (i.i.d.) exponential random variables with a unit mean since an i.i.d. Rayleigh fading channel model is assumed. Although different mean values can reflect the effects of path-loss, the unit mean assumption simplifies analysis and efficiently delivers key intuitions. We do not take into account primary transmitters here because the focus of this paper is on the capacity of the secondary network. Each primary receiver imposes its interference constraint on the secondary transmitters for reliable communication regardless of data exchanges in the secondary network. The interference temperature, maximum allowable interference level perceived at each primary receiver, is denoted by $Q$. Secondary transmitters adapt their transmit power according to the interference channel gains between secondary transmitters and primary receivers to comply with the interference regulation at the primary receivers. Correspondingly, each secondary transmitter is required to attain the knowledge of interference channel gains by periodically sensing pilot signal from the primary receiver assuming the channel reciprocity [11]. Despite the burden on the primary users, spectrum sharing based on the interference temperature can more aggressively improve spectral efficiency than other cognitive radio techniques [12]. For medium access control among secondary transmitters, opportunistic user selection is adopted based on practical requirements of complexity and the amount of feedback. A secondary transmitter with the best signal channel gain among those not violating the interference regulation is selected for transmission.

Fig. 2 shows a slot structure for sensing and data transmission at the secondary transmitters. At each sensing period, $m$ secondary transmitters among $N_{s}$ secondary transmitters

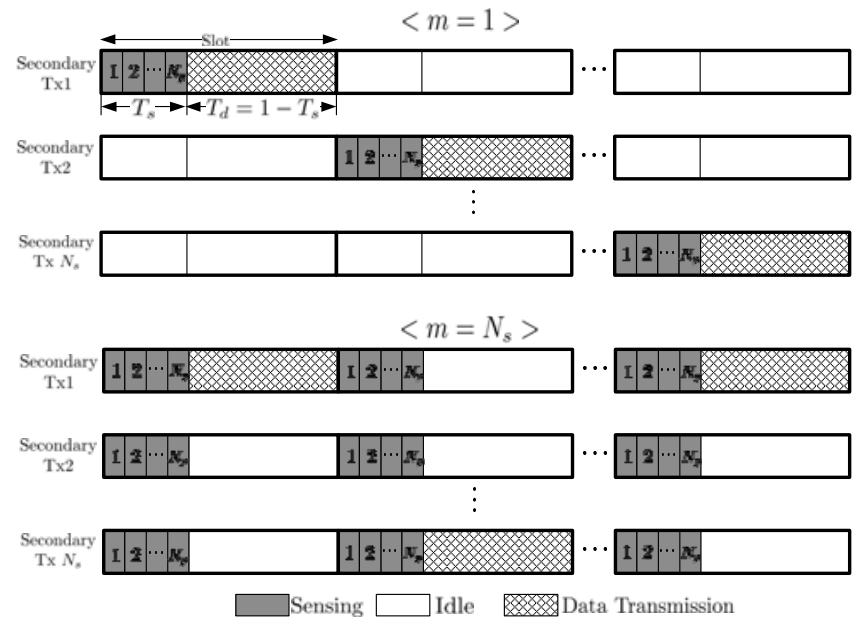

Fig. 2. Slot structure when $m$ secondary transmitters perform sensing.

estimate the interference channel gains. The term $t_{s}$ denotes the required time duration for reliable sensing of an interference channel [13]. Since there are $N_{p}$ primary receivers, a secondary transmitter devotes $T_{s}=N_{p} t_{s}$ in sensing at each sensing period. Every sensing period is followed by the time duration for data transmission, $T_{d}$. That is, a slot consists of $T_{s}$ and $T_{d}$ as shown in Fig. 2. For simplicity, the slot length is assumed to be $T_{s}+T_{d}=1$. The total power consumption during the sensing period depends on $m$. As $m$ decreases, the total power consumption decreases owing to a longer sensing interval but the multiuser diversity gain decreases since the secondary transmitters not performing sensing are excluded in the opportunistic user selection for a given slot. There is a fundamental tradeoff between power consumption and multiuser diversity gain.

\section{Average Achievable Capacity Normalized by ENERGY CONSUMPTION}

Since the power consumption in spectrum sensing at the secondary transmitters is neglected in the conventional capacity analysis of spectrum sharing systems, the average capacity of spectrum sharing systems is overestimated. Therefore, we derive a new metric, achievable average capacity normalized by energy consumption to reflect both time and power overhead due to spectrum sensing in achievable capacity. The achievable capacity normalized by energy consumption represents the energy efficiency in achieving the capacity.

In each slot, $m$ secondary transmitters among $N_{s}$ secondary transmitters perform sensing to estimate the interference channel gains and $\mathcal{S}_{m}$ denotes a set consisting of the secondary transmitters performing sensing. The cardinality of $\mathcal{S}_{m}$ is obviously $\left|S_{m}\right|=m$. The power consumption by estimating $N_{p}$ interference channels at a secondary transmitter during $T_{s}$ is $\lambda_{p} P$ where $P$ is a power constraint at a secondary transmitter and $\lambda_{p}$ represents the proportion of power, $0<\lambda_{p}<1$. On the other hand, in the slots where a secondary transmitter does not perform sensing, there is no power consumption for sensing and data transmission. Since $m$ secondary transmitters perform sensing and one secondary transmitter is selected for transmission among the $m$ sensing secondary transmitters, the 
total energy consumption in a system during a slot interval is given by

$$
E(m)=m T_{s} \lambda_{p} P+T_{d} P .
$$

Then, the regulated transmit power from the $i$-th secondary transmitter in the set, $\mathcal{S}_{m}$, for given $m$ is obtained by

$$
P_{i}=\left\{\begin{array}{cc}
P, & P \alpha_{i} \leq Q \\
\frac{Q}{\alpha_{i}}, & P \alpha_{i}>Q
\end{array}, \quad i \in \mathcal{S}_{m}\right.
$$

where $\alpha_{i}$ denotes an effective interference channel gain of $i$-th secondary transmitter defined as

$$
\alpha_{i} \triangleq \max _{1 \leq j \leq N_{p}} \alpha_{i, j}
$$

whose probability density function (PDF) is given by

$$
f_{\alpha_{i}}(x)=N_{p} e^{-x}\left(1-e^{-x}\right)^{N_{p}-1}
$$

since $\alpha_{i, j}$ are i.i.d. exponential random variables. Correspondingly, the received SNR from the $i$-th secondary transmitter $\left(i \in \mathcal{S}_{m}\right)$ is obtained by

$$
\gamma_{i} \triangleq \frac{P_{i} \beta_{i}}{\sigma^{2}}=P_{i} \beta_{i}
$$

where $\sigma^{2}$ denotes the variance of white Gaussian noise and is assumed to be 1 so that the transmit power can be also considered as the transmit SNR. $\beta_{i}$ is the $i$-th signal channel gain between the $i$-th secondary transmitter and the secondary receiver. With opportunistic user selection among secondary transmitters in $\mathcal{S}_{m}$, the received SNR at the secondary receiver is given by

$$
\gamma_{\max \mid \mathcal{S}_{m}} \triangleq \max _{i \in \mathcal{S}_{m}} \gamma_{i}
$$

since the secondary transmitter with the highest $\gamma_{i}$ is selected for transmission in the set, $\mathcal{S}_{m}$. For given $m$ and $\mathcal{S}_{m}$, the achievable capacity is obtained by

$$
C\left(\mathcal{S}_{m}\right)=\frac{T_{d}}{T_{d}+T_{s}} \log _{2}\left(1+\gamma_{\max \mid \mathcal{S}_{m}}\right)
$$

where the scaling term $T_{d} /\left(T_{d}+T_{s}\right)$ reflects a throughput loss due to the required time for sensing. Since each secondary transmitter is included in the sensing set or not, the optimal sensing set is determined among $2^{N_{s}}$ possible sensing sets. Therefore, the optimal average capacity normalized by energy consumption of the spectrum sharing system with opportunistic user selection is obtained as

$$
\begin{aligned}
C_{\text {norm }}^{\text {opt }} & \triangleq \mathbb{E}\left[\max _{1 \leq m \leq N_{s}} \frac{\max _{\mathcal{S}_{m}} C\left(\mathcal{S}_{m}\right)}{E(m)}\right] \\
& =\mathbb{E}\left[\max _{1 \leq m \leq N_{s}} \frac{\max _{\mathcal{S}_{m}} C\left(\mathcal{S}_{m}\right)}{m T_{s} \lambda_{p} P+T_{d} P}\right] .
\end{aligned}
$$

Although Eq. (8) does not have a closed-form solution, we can obtain the solution using an exhaustive search algorithm. However, it is not practically feasible to achieve the optimal normalized capacity since it requires a priori knowledge of the effective interference channel gains for secondary transmitters even not in the optimal sensing set. In addition, computational complexity in finding the optimal capacity exponentially increases as the number of secondary transmitter increases.

\section{A NeAR-OPtimal Solution}

In this section, we propose a simple and practical suboptimal scheme approaching the optimal strategy. In the proposed scheme, a secondary receiver selects $n$ secondary transmitters with $n$ highest signal channel gains to perform sensing at each slot. The ordered gains are indexed by $\hat{i}$ such as $\beta_{\widehat{1}} \geq \beta_{\widehat{2}} \geq \cdots \geq \beta_{\widehat{N}_{s}}$ for a given slot. Since only $n$ secondary transmitters from the ordered index 1 to $n$ perform sensing in the slot, the energy consumption in each slot is given by

$$
E(n)=n T_{s} \lambda_{p} P+T_{d} P .
$$

Similarly to Eq. (2), the regulated transmit power from the $\widehat{i}$ th secondary transmitter whose signal channel gain is ranked within the top $n$ among $N_{s}$ secondary transmitters is given by

$$
P_{\widehat{i}}=\left\{\begin{array}{cc}
P, & P \alpha_{\hat{i}} \leq Q \\
\frac{Q}{\alpha_{\hat{i}}}, & P \alpha_{\hat{i}}>Q
\end{array} \quad \widehat{i} \in\{1,2, \cdots, n\}\right.
$$

and the corresponding received SNR at the secondary receiver from the $\hat{i}$-th secondary transmitter is given by

$$
\gamma_{\hat{i}}=P_{\hat{i}} \beta_{\hat{i}} .
$$

Since only $n$ secondary transmitters are candidates of opportunistic user selection, the received SNR at the secondary receiver by opportunistic user selection is given by

$$
\gamma_{\max }^{\text {best }-n} \triangleq \max _{\widehat{i} \in\{1,2, \cdots, n\}} \gamma_{\widehat{i}} .
$$

Then, the average normalized capacity of the proposed scheme is obtained by

$$
\begin{aligned}
C_{\text {norm }}^{\text {best-n }} & =\mathbb{E}\left[\frac{\frac{T_{d}}{\left(T_{d}+T_{s}\right)} \log _{2}\left(1+\gamma_{\text {max }}^{\text {best-n }}\right)}{E(n)}\right] \\
& =\frac{T_{d}}{n T_{s} \lambda_{p} P+T_{d} P} \mathbb{E}\left[\log _{2}\left(1+\gamma_{\text {max }}^{\text {best-n }}\right)\right] .
\end{aligned}
$$

Compared to the optimal scheme in Eq. (8), the proposed scheme does not require prior information of interference channels to decide a sensing set and avoids the exhaustive search over $\left\{\mathcal{S}_{m}\right\}$ and $m$, but it approaches the optimal scheme in terms of normalized capacity. Even though a closed form result for Eq. (13) is not available for a general value of $n$, we can derive semi-closed form solutions for $n=1$ and $n=N_{s}$, from which we can more intuitively understand the effects of key parameters.

For $n=1$, the received SNR at the secondary receiver, $\gamma_{\max }^{\text {best-1 }}$, is given by

$$
\gamma_{\max }^{\text {best }-1}=\gamma_{\widehat{1}}=P_{\widehat{1}} \beta_{\widehat{1}},
$$

where the PDF of $\beta_{\widehat{1}}$ is given by

$$
f_{\beta_{\hat{1}}}(x)=N_{s} e^{-x}\left(1-e^{-x}\right)^{N_{s}-1} .
$$


The PDF of $\gamma_{\max }^{\text {best-1 }}$ is obtained in Appendix A as

$$
\begin{aligned}
& f_{\gamma_{\max }^{\text {best }}}(x) \\
& =\left(1-e^{-\frac{Q}{P}}\right)^{N_{p}} \frac{N_{s}}{P} \sum_{k=1}^{N_{s}}\left(\begin{array}{c}
N_{s}-1 \\
k-1
\end{array}\right)(-1)^{k-1} e^{-\frac{k x}{P}} \\
& +\frac{Q}{P} N_{s} N_{p} \sum_{k=1}^{N_{s}}\left(\begin{array}{c}
N_{s}-1 \\
k-1
\end{array}\right)(-1)^{k-1} \\
& \times \sum_{j=1}^{N_{p}}\left(\begin{array}{c}
N_{p}-1 \\
j-1
\end{array}\right)(-1)^{j-1} e^{-j \frac{Q}{P}} e^{-\frac{k x}{P}} \frac{j Q+P+k x}{(j Q+k x)^{2}} .
\end{aligned}
$$

Using Eqs. (13) and (16), the average normalized capacity of the proposed best $n$ scheme for $n=1$ is obtained as

$$
\begin{aligned}
& C_{\text {norm }}^{\text {best-1 }}=\frac{T_{d}}{T_{s} \lambda_{p} P+T_{d} P} \mathbb{E}\left[\log _{2}\left(1+\gamma_{\text {max }}^{\text {best }-1}\right)\right] \\
& =\frac{T_{d}}{T_{s} \lambda_{p} P+T_{d} P} \int_{0}^{\infty} \log _{2}(1+x) f_{\gamma_{\text {max }}^{\text {best-1 }}}(x) d x \\
& =\frac{T_{d}}{T_{s} \lambda_{p} P+T_{d} P}\left(1-e^{-\frac{Q}{P}}\right)^{N_{p}} \frac{N_{s}}{P} \\
& \times \sum_{k=1}^{N_{s}}\left(\begin{array}{c}
N_{s}-1 \\
k-1
\end{array}\right)(-1)^{k-1} \frac{P e^{\frac{k}{P}} E_{1}\left(\frac{k}{P}\right)}{k \ln (2)} \\
& +\frac{T_{d}}{T_{s} \lambda_{p} P+T_{d} P} \frac{Q N_{s} N_{p}}{P} \sum_{k=1}^{N_{s}}\left(\begin{array}{c}
N_{s}-1 \\
k-1
\end{array}\right) \\
& \times(-1)^{k-1} \sum_{j=1}^{N_{p}}\left(\begin{array}{c}
N_{p}-1 \\
j-1
\end{array}\right)(-1)^{j-1} e^{-j \frac{Q}{P}} P \\
& \times \frac{e^{j \frac{Q}{P}} E_{1}\left(j \frac{Q}{P}\right)-e^{\frac{k}{P}} E_{1}\left(\frac{k}{P}\right)}{\ln (2) k(k-j Q)} \\
& =\frac{T_{d}}{T_{s} \lambda_{p} P+T_{d} P}\left(1-e^{-\frac{Q}{P}}\right)^{N_{p}} \frac{N_{s}}{\ln (2)} \\
& \times \sum_{k=1}^{N_{s}}\left(\begin{array}{c}
N_{s}-1 \\
k-1
\end{array}\right) \frac{(-1)^{k-1}}{k} e^{\frac{k}{P}} E_{1}\left(\frac{k}{P}\right) \\
& +\frac{T_{d}}{T_{s} \lambda_{p} P+T_{d} P} \frac{Q N_{s} N_{p}}{\ln (2)} \sum_{k=1}^{N_{s}}\left(\begin{array}{c}
N_{s}-1 \\
k-1
\end{array}\right) \\
& \times \frac{(-1)^{k-1}}{k} \sum_{j=1}^{N_{p}}\left(\begin{array}{c}
N_{p}-1 \\
j-1
\end{array}\right)(-1)^{j-1} \\
& \times \frac{E_{1}\left(j \frac{Q}{P}\right)-e^{\frac{k-j Q}{P}} E_{1}\left(\frac{k}{P}\right)}{k-j Q},
\end{aligned}
$$

where $E_{1}(\cdot)$ denotes an exponential integral function.

On the other hand, for $n=N_{s}$, we can derive the average normalized capacity can be also described as

$$
\begin{aligned}
C_{\text {norm }}^{\text {best-N }} & =\frac{T_{d} \mathbb{E}\left[\log _{2}\left(1+\gamma_{\text {max }}^{\text {best-Ns }}\right)\right]}{N_{s} T_{s} \lambda_{p} P+T_{d} P} \\
& =\frac{T_{d} \int_{0}^{\infty} \log _{2}(1+x) f_{\gamma_{\text {max }}^{\text {best }} \text { s }}(x) d x}{N_{s} T_{s} \lambda_{p} P+T_{d} P} \\
& =\frac{T_{d} \int_{0}^{\infty} \log _{2}(1+x) N_{s} f_{\gamma_{i}}(x)\left(F_{\gamma_{i}}(x)\right)^{N_{s}-1} d x}{N_{s} T_{s} \lambda_{p} P+T_{d} P},
\end{aligned}
$$

where $f_{\gamma_{i}}(x)$ and $F_{\gamma_{i}}(x)$ are given, respectively, by [7]

$$
\begin{aligned}
f_{\gamma_{i}}(x)= & \frac{1}{P}\left(1-e^{-\frac{Q}{P}}\right)^{N_{p}} e^{-\frac{x}{P}} \\
& +\frac{N_{p} Q}{P} e^{-\frac{x}{P}} \sum_{k=1}^{N_{p}}\left(\begin{array}{c}
N_{p}-1 \\
k-1
\end{array}\right)(-1)^{k-1} \\
& \times e^{-k \frac{Q}{P}} \frac{k Q+P+x}{(k Q+x)^{2}} \\
F_{\gamma_{i}}(x)= & \left(1-e^{-\frac{Q}{P}}\right)^{N_{p}}\left(1-e^{-\frac{x}{P}}\right) \\
& +N_{p} \sum_{k=1}^{N_{p}}\left(\begin{array}{c}
N_{p}-1 \\
k-1
\end{array}\right)(-1)^{k-1} \\
& \times e^{-k \frac{Q}{P}}\left[\frac{1}{k}-\frac{Q}{Q k+x} e^{-\frac{x}{P}}\right] .
\end{aligned}
$$

It should be noted that the index $i$ is used instead of the ordered index $\hat{i}$ since all the secondary transmitters are candidates of opportunistic user selection.

\section{Numerical RESUlts}

Fig. 3(a) and Fig. 3(b) show the capacity (before normalization by energy consumption) and normalized capacity, respectively, for varying available power-to-noise power ratios of secondary transmitters when $N_{s}=10, N_{p}=5, Q=0 \mathrm{~dB}$, $\lambda_{p}=0.8$, and $t_{s}=0.05$. If the normalization by energy consumption is not taken into account, the best- $N_{s}$ scheme achieves the optimal capacity even in the region where the peak power (or transmit SNR) is much higher than interference temperature $Q$ as shown in Fig. 3(a). In the high transmit power region, the effect of interference temperature regulation is distinct. Therefore, it is beneficial that all the secondary transmitters participate in sensing and opportunistic transmit selection. On the other hand, the best-1 scheme achieves almost the same capacity as the optimal scheme and the best$N_{s}$ scheme in the low transmit power region because the effect of interference channel is not severe in the low transmit power region and, hence, the selection based on signal channel gains becomes crucial.

If the energy consumption due to spectrum sensing is reflected in capacity evaluation, the results are different. Fig. 3(b) shows the average capacity normalized by the total energy consumed in both sensing and data transmission. Contrary to Fig. 3(a), the normalized average capacity decreases with the transmit power because the energy consumption is proportional to the transmit power, while the capacity improves in a logarithmic scale as the transmit power increases. It should be also noted from this figure that the best- 1 scheme rather approaches the optimal scheme. In the low transmit power region where the effect of interference constraints is not significant, the best- 1 scheme achieves almost the same normalized average capacity as the optimal one. Even in the high transmit power region where the interference constraints mainly affect the selection of a secondary transmitter, the best1 scheme approaches the optimal scheme only with a small gap. These results indicate that the best- 1 scheme is a near optimal scheme in the context of energy efficiency.

Fig. 4(a) and Fig. 4(b) show the capacity and normalized capacity versus interference temperature $Q$ when $P=0 \mathrm{~dB}$, 


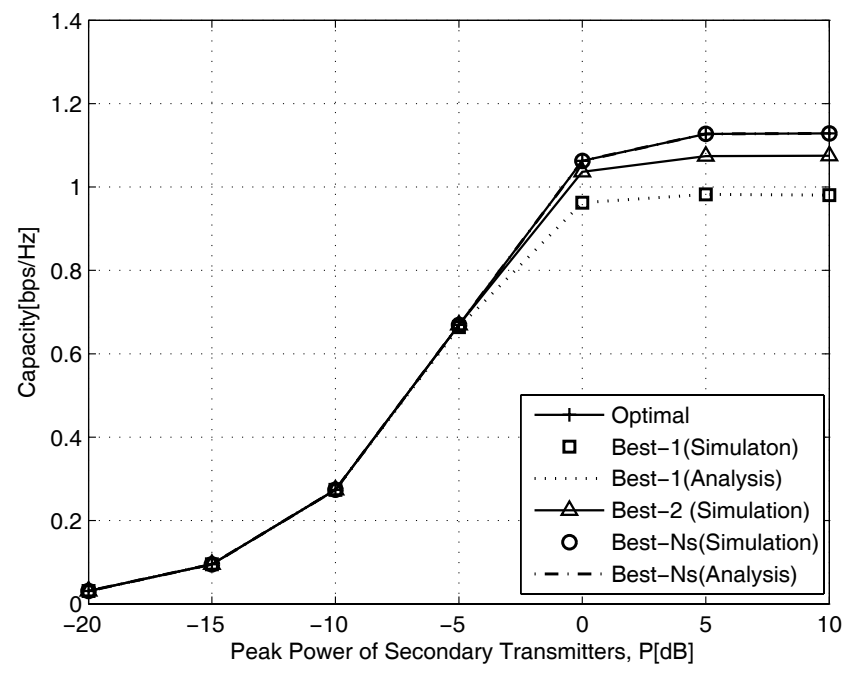

(a) Capacity (before normalization by consumed energy)

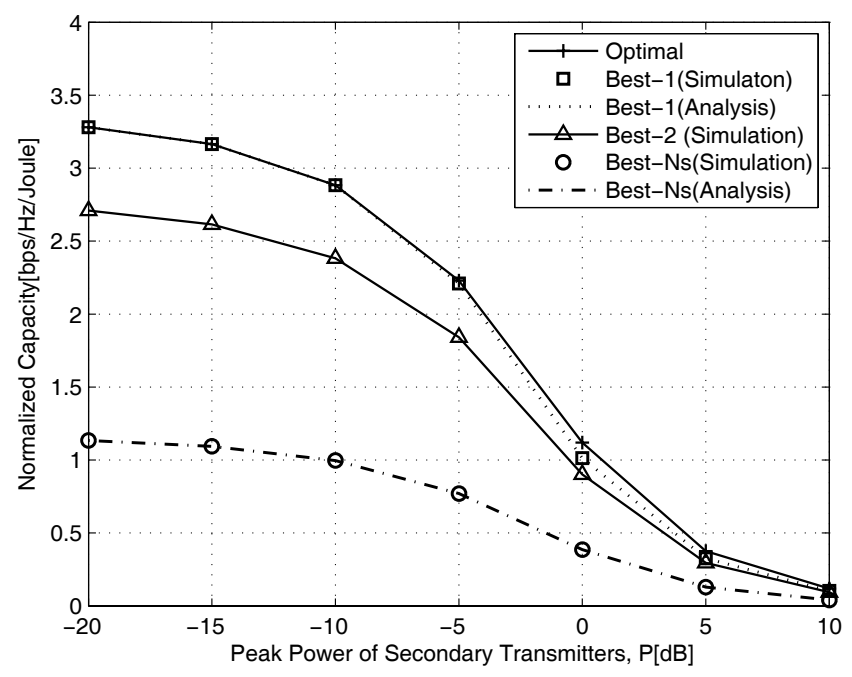

(b) Normalized capacity

Fig. 3. Capacity and normalized capacity versus $P . Q=0 \mathrm{~dB}, N_{s}=10$, $N_{p}=5, \lambda_{p}=0.8$, and $t_{s}=0.05$. The number of simulation iterations is set to 20,000 .

respectively. Fig. 4(a) indicates that without normalization by the energy consumption, the best- $N_{s}$ scheme achieves the optimal capacity in all regions, while the best- 1 scheme approaches the optimal capacity only in the region where $Q$ is relatively high compared to $P$. On the other hand, if the energy consumption is taken into account, the best- 1 scheme approaches the optimal normalized capacity in the high $Q$ region as shown in Fig. 4(b) because the best- 1 scheme consumes less sensing power compared to the best- $N_{s}$ scheme. Fig 4(a) and Fig. 4(b) also show that both the capacity and normalized capacity increase with $Q$ because $\operatorname{larger} Q$ value allows more opportunistic utilization of transmit power at the secondary transmitters.

\section{CONCLUSION}

We defined and derived the normalized capacity of spectrum sharing systems with opportunistic user selection to properly

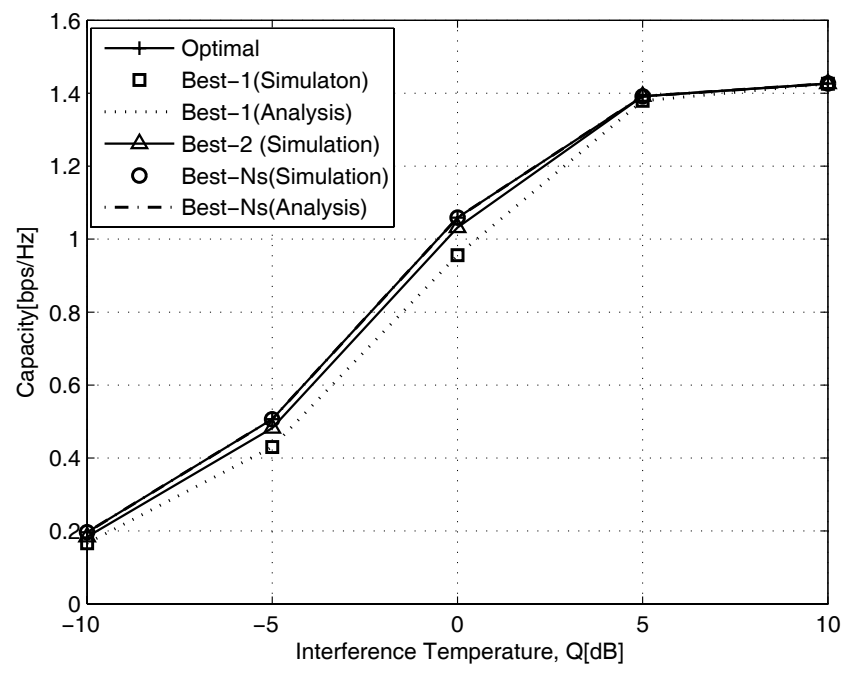

(a) Capacity (before normalization by consumed energy)

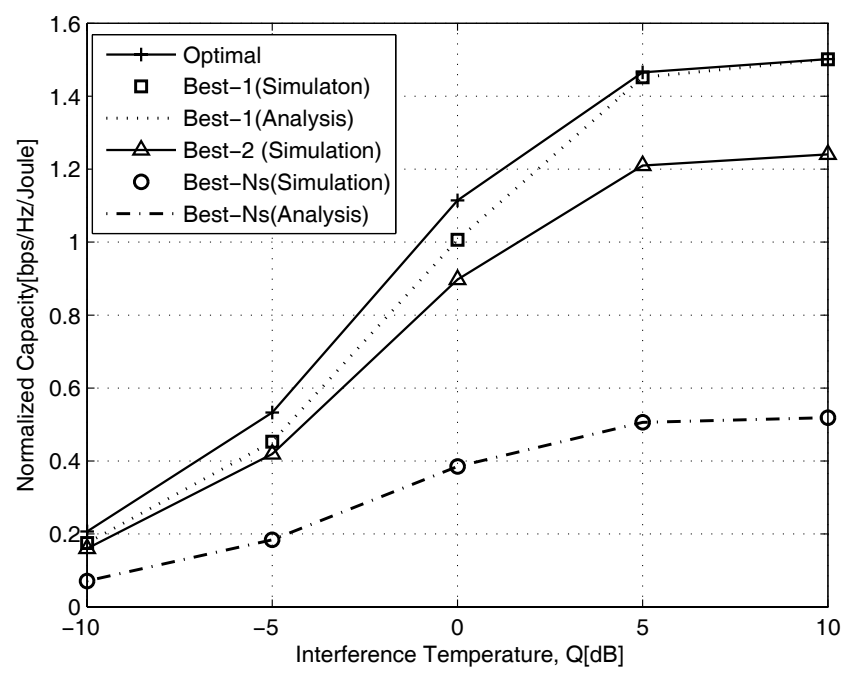

(b) Normalized capacity

Fig. 4. Capacity and normalized capacity versus $Q . P=0 \mathrm{~dB}, N_{s}=10$, $N_{p}=5, \lambda_{p}=0.8$, and $t_{s}=0.05$. The number of simulation iterations is set to 20,000

incorporate time and energy overhead of spectrum sensing in capacity evaluation. Our analytical and numerical results show that in spectrum sharing environments, a policy that all secondary transmitters sense their interference channels is not optimal any more in the context of energy efficiency because that policy consumes too much energy in spectrum sensing. The optimal normalized capacity was evaluated based on the developed formula. To circumvent the infeasibility and high computational complexity of the optimal strategy, we proposed a simple and practical best $n$ scheme and showed that the best- 1 scheme substantially approaches the optimal strategy in terms of normalized capacity especially when the effects of interference constraints are not distinct. 


\section{APPENDIX A}

DERIVATION OF $f_{\gamma_{\text {max }}^{\text {best-1 }}}(x)$ FOR THE BEST- 1 SCHEME

Let $y=\beta_{\widehat{1}}$ and $z=\alpha_{\hat{1}}$. Then, using the total probability theory [14], the PDF of $\gamma_{\max }^{b e s t-1}$ is obtained as

$$
\begin{aligned}
& f_{\gamma_{\text {max }}^{\text {best } 1}}(x) \\
= & \left(1-e^{-\frac{Q}{P}}\right)^{N_{p}} \frac{1}{P} f_{y}\left(\frac{x}{P}\right) \\
& +\frac{1}{Q} \int_{\frac{Q}{P}}^{\infty} z f_{y}\left(\frac{x z}{Q}\right) f_{z}(z) d z \\
= & \left(1-e^{-\frac{Q}{P}}\right)^{N_{p}} \frac{N_{s}}{P} \sum_{k=1}^{N_{s}}\left(\begin{array}{c}
N_{s}-1 \\
k-1
\end{array}\right)(-1)^{k-1} e^{-\frac{k x}{P}} \\
& +\frac{1}{Q} \int_{\frac{Q}{P}}^{\infty} z f_{y}\left(\frac{x z}{Q}\right) f_{z}(z) d z,
\end{aligned}
$$

where the second equality comes from $f_{y}(y)=N_{s} e^{-y}(1-$ $\left.e^{-y}\right)^{N_{s}-1}$ and binomial expansion. The second term in Eq. (A.1) is derived as

$$
\begin{aligned}
& \frac{1}{Q} \int_{\frac{Q}{P}}^{\infty} z f_{y}\left(\frac{x z}{Q}\right) f_{z}(z) d z \\
& =\frac{N_{s} N_{p}}{Q} \int_{\frac{Q}{P}}^{\infty} z e^{-\frac{x z}{Q}}\left(1-e^{-\frac{x z}{Q}}\right)^{N_{s}-1} \\
& \times e^{-z}\left(1-e^{-z}\right)^{N_{p}-1} d z \\
& =\frac{N_{s} N_{p}}{Q} \int_{\frac{Q}{P}}^{\infty} z \sum_{k=1}^{N_{s}}\left(\begin{array}{c}
N_{s}-1 \\
k-1
\end{array}\right)(-1)^{k-1} \\
& \times e^{-\frac{k x z}{Q}} \sum_{j=1}^{N_{p}}\left(\begin{array}{c}
N_{p}-1 \\
j-1
\end{array}\right)(-1)^{j-1} e^{-j z} d z \\
& =\frac{N_{s} N_{p}}{Q} \sum_{k=1}^{N_{s}}\left(\begin{array}{c}
N_{s}-1 \\
k-1
\end{array}\right)(-1)^{k-1} \sum_{j=1}^{N_{p}}\left(\begin{array}{c}
N_{p}-1 \\
j-1
\end{array}\right)(-1)^{j-1} \\
& \times \int_{\frac{Q}{P}}^{\infty} z e^{-z\left(j+\frac{k x}{Q}\right)} d z \\
& =\frac{Q}{P} N_{s} N_{p} \sum_{k=1}^{N_{s}}\left(\begin{array}{c}
N_{s}-1 \\
k-1
\end{array}\right)(-1)^{k-1}
\end{aligned}
$$

$$
\times \sum_{j=1}^{N_{p}}\left(\begin{array}{c}
N_{p}-1 \\
j-1
\end{array}\right)(-1)^{j-1} e^{-j \frac{Q}{P}} e^{-\frac{k x}{P}} \frac{j Q+P+k x}{(j Q+k x)^{2}}
$$

where the first equality is derived from binomial expansion.

\section{REFERENCES}

[1] Federal Communications Commision, "Spectrum policy task force report, ET Docket No. 02-135," Nov. 2002.

[2] A. Petrin and P. Steffes, "Analysis and comparison of spectrum measurements performed in urban and rural areas to determine the total amount of spectrum usage," in Proc. ISART, pp. 9-12, Mar. 2005.

[3] D. Cabric, I. D.O'Donnell, M. S.-W. Chen, and R. W. Brodersen, "Spectrum sharing radios," IEEE Circuits Systems Mag., vol. 6, no. 2, pp. 30-45, 2006.

[4] M. Gastpar, "On capacity under receiver and spatial spectrum-sharing constraints," IEEE Trans. Inform. Theory, vol. 53, no. 2, pp. 471-487, Feb. 2007.

[5] A. Ghasemi and E. S. Sousa, "Fundamental limits of spectrum-sharing in fading environments," IEEE Trans. Wireless Commun., vol. 6, no. 2, pp. 649-658, Feb. 2007.

[6] R. Zhang and Y.-C. Liang, "Exploiting multi-antennas for opportunistic spectrum sharing in cognitive radio networks," IEEE J. Select. Topics Signal Processing, vol. 2, no. 1, pp. 88-102, Feb. 2008.

[7] T. W. Ban, D. K. Sung, B. C. Jung, and W. Choi, "Capacity analysis of an opportunistic scheduling system in a spectrum sharing environment," in Proc. IEEE Globecom, Nov. 2008.

[8] T. W. Ban, W. Choi, B. C. Jung, and D. K. Sung, "Multiuser diversity in a spectrum sharing system," IEEE Trans. Wireless Commun., vol. 8, no. 1, pp. 102-106, Jan. 2009.

[9] D. Cabric, A. Tkachenko, and R. Brodersen, "Experimental study of spectrum sensing based on energy detection and netwok cooperation," in Proc. 2nd Annual International Wireless Internet Conf. (WICON), TAPAS Workshop, Aug. 2006.

[10] A. Ghasemi and E. Sousa, "Optimization of spectrum sensing for opportunistic spectrum access in cognitive radio networks," in Proc. IEEE CCNC, pp. 1022-1026, Jan. 2007.

[11] Q. Zhao, S. Geirhofer, L. Tong, and B. Sadler, "Opportunistic spectrum access via periodic channel sensing," IEEE Trans. Signal Processing, vol. 56, no. 2, pp. 785-796, Feb. 2008.

[12] Website of FCC, [Online]. Available: http://www.fcc.gov/sptf/reports.html.

[13] Y. Chen, Q. Zhao, and A. Swami, "Joint design and separation principle for opportunistic spectrum access in the presence of sensing errors," IEEE Trans. Inform. Theory, vol. 54, no. 5, pp. 2053-2071, May 2008.

[14] A. Papoulis and S. U. Pillai, Probability, Random Variables and Stochastic Processes, 4th ed. McGraw-Hill, 2002. 